\title{
A community journal for an evolving field
}

\author{
Today, we are proud to launch Nature Metabolism, a new dedicated home for exciting research and commentary \\ that spans the spectrum of metabolism research.
}

R esearch in the fields of cellular and systemic metabolism has flourished in recent years, providing fundamental new insights into the inner workings of cells and enhancing the understanding of some of the world's most common diseases. While Nature and its sister titles have a long history of publishing metabolism research, the steady rise in innovative high-quality and high-impact metabolism research has prompted us to launch a new journal specifically targeted at the wider metabolism community.

Our scope is broad and built around the central themes of metabolism and homeostasis. Research published in Nature Metabolism will shed new light on how cellular metabolism informs cellular function, the biology of metabolic organs, how metabolic dysfunction can lead to disease and the treatment of metabolic diseases. Touching on the physiological principle of homeostasis, our journal will also provide a forum for papers that are important conceptual advances in the molecular understanding of organismal physiology.

By capturing the full breadth of metabolism research-from fundamental cell biology to biomedical and translational research-Nature Metabolism will create unique points of synergy and foster the exchange of ideas between researchers working in the various subdisciplines covered in our pages. As a community journal, we provide a forum for lively debate and lend the community a voice that, if needed, will reach beyond our core audience to advocate on their behalf. Our role as a community champion extends to the service we offer to our authors and reviewers: we want to be a reliable partner in the publishing process, not simply gatekeepers. As a journal, we strive to provide you with a direct line of communication to our editors and a fair and transparent decision-making process, including clear guidance when inviting revised manuscripts.

As expected from a Nature Research journal, our objective is to publish the most significant research in our field-exciting, thought provoking, conceptually novel work that opens doors to new areas of research or answers long-standing questions. This goal cannot be achieved without the support of the scientific community that we serve. We do not take this support for granted and are thus immensely grateful to the authors and reviewers who worked with us at a time when our journal had not yet published any content.

Today we are excited to share with you the first full issue of Nature Metabolism. The variety and quality of the content in this first issue sets a great example of what we would like the journal to be: inclusive, broad, highquality and timely. Clicking through our virtual pages, you will find articles providing new insights into cellular metabolism; vascular, bone and adipose tissue physiology; and cholesterol metabolism, insulin resistance and cancer. We also present our first two Review articles, one about non-metabolic signalling functions for tricarboxylic acid cycle intermediates and the other on mechanisms through which the microbiota regulates organismal energy homeostasis. Illustrating the role of Nature Metabolism as a community journal is a Comment raising awareness of variation among mouse substrains used in research. We hope you will enjoy reading these articles, and the accompanying News \& Views, as much as we enjoyed working with our authors to get them published. Finally, the birth of a new journal also offers an opportunity to think about where the field is heading. To find out, we asked ten experts to chart the future of research in their areas for us.

Rapid technological development in key areas such as sequencing, imaging and machine learning is a clear sign that the field continues to evolve and that the future of metabolism research is bright. The launch of Nature Metabolism today signals our commitment to supporting the growing metabolism research community for years to come and to shaping scientific progress in the field by highlighting some of the most important advances it produces. We invite you to join us on this exciting journey!

Published online: 7 January 2019 https://doi.org/10.1038/s42255-018-0026-3 